\title{
In praise of the impact factor
}

\author{
John Antonakis \\ University of Lausanne \\ Nicolas Bastardoz \\ University of Zurich \\ Philippe Jacquart \\ EMLYON Business School
}

\begin{abstract}
The impact factor has been criticized on several fronts, including that the distribution of citations to journal articles is heavily skewed. We nuance these critiques and show that the number of citations an article receives is significantly predicted by journal impact factor. Thus, impact factor can be used as a reasonably good proxy of article quality.

Introduction: We acknowledge that the journal impact factor (JIF) is a limited metric (Callaway, 2016); it should not be used as the sole criterion to judge the quality of a particular article. Why? Briefly, citations articles receive are not equally distributed; some articles are very heavily cited whereas many are not. Typically, a few very highly cited articles appear to drive the impact factor of a journal. Thus, judging quality of a particular article published in the journal using the journal impact factor is something that many find unjustifiable; such critics suggest that it is folly to use JIFs as a gauge of article quality or the future citations of the article. We disagree.
\end{abstract}

Design: We report on data published in Antonakis, Bastardoz, Jacquart, \& Shamir (2016), who gathered data on $\mathbf{2 7 6}$ articles nested in 49 journals. The key predictor we wish to showcase here is journal impact factor, while controlling for article age and its quadratic effect (to account for article decay over time), prestige of university of authors, number of authors, field impact factor, number of cited references, number of authors, and article type. We used a 5-year average impact factor given that journal impact factor rank is very stable over time; indeed, over a 10-year period the intraclass correlation coefficient of all journals in the Web of Science categories of Applied Psychology, Business, Management, and Social Psychology is .90.

Results: Although the distribution of citations to articles is heavily skewed, as explained by a power law (Antonakis, Bastardoz, Liu, \& Schriesheim, 2014), there is a still a very strong relationship between the impact factor and the citations an article in the journal may receive. On a bivariate level, the number of article citations received per year is quite strongly correlated with the 5-year JIF, $r(276)=.60$, as it the correlation with total citations, $r(276)=.49$ (see Table 13 in on-line supplementary materials in Antonakis et al., 2016). Given that the outcome is a count and heavily dispersed, using a negative binomial regression, we found that the JIF predicts citations received per year, even when controlling for the aforementioned covariates. (see Table 1 in on-line supplementary materials, Antonakis et al., 2016). The effect of the JIF is rather large. The incidence response ratio is 1.27; specifically, an increase of JIF by 1 unit increases the citations an article receives by $26.98 \%$. 
The figure below shows that, ceteris paribus, getting an article published in a higher impact factor journal means more citations for the article.

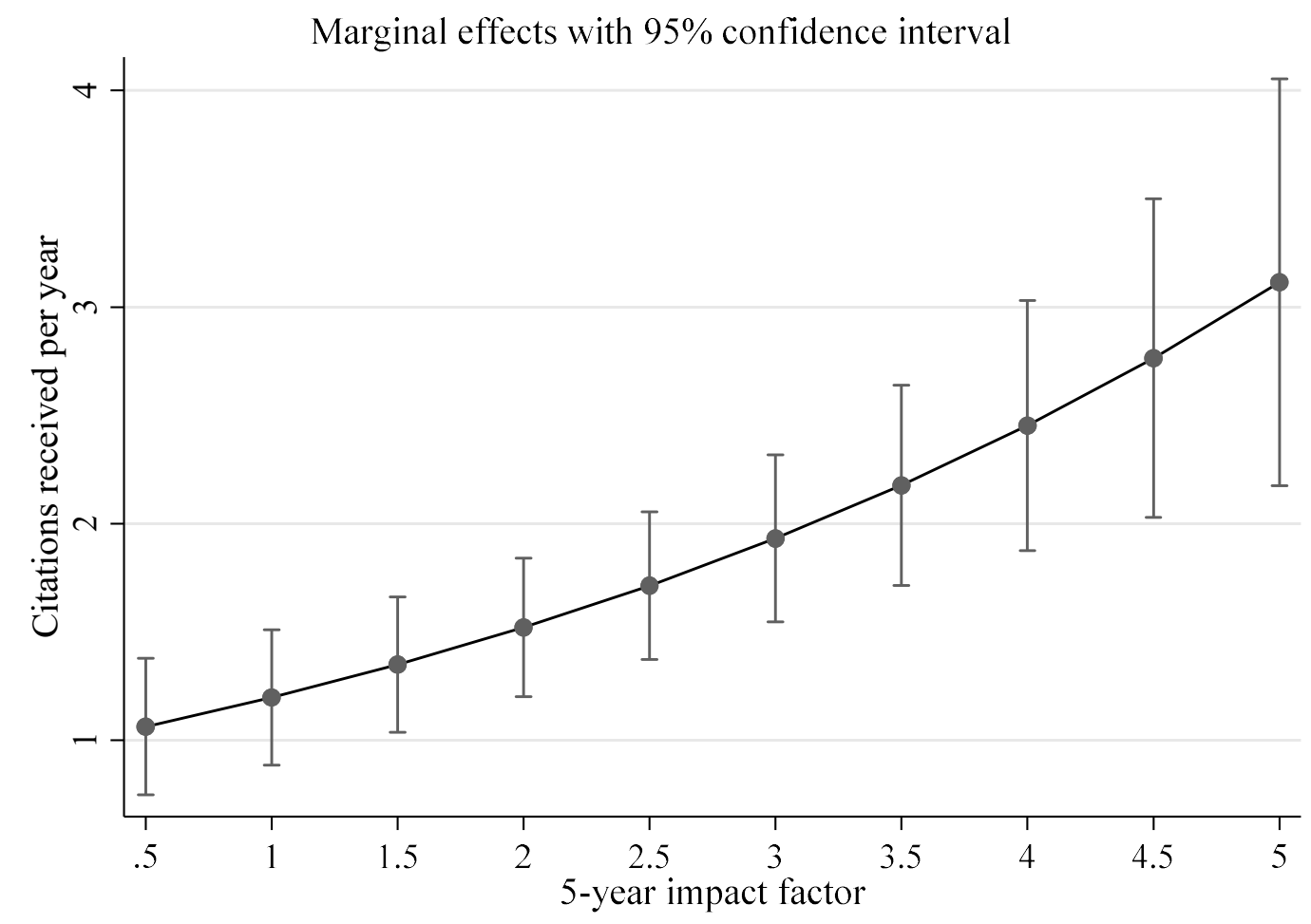

Figure 1: Predicting citations received per year

Results still hold when predicting total citations received.

Conclusions: Previous research has shown that articles published in higher impact factor journals tend to be more cited (Larivière \& Gingras, 2010). Of course, the JIF cannot replace expert judgement; but it is a valid cue of article quality.

\section{References}

Antonakis, J., Bastardoz, N., Jacquart, P., \& Shamir, B. 2016. Charisma: An ill-defined and ill-measured gift. Annual Review of Organizational Psychology and Organizational Behavior, 3(1): 293-319. Antonakis, J., Bastardoz, N., Liu, Y., \& Schriesheim, C. A. 2014. What makes articles highly cited? The Leadership Quarterly, 25(1): 152-179.

Callaway, E. 2016. Publishing elite turns against impact factor. Nature, 535(7611): 210-211.

Larivière, V., \& Gingras, Y. 2010. The impact factor's Matthew Effect: A natural experiment in bibliometrics. Journal of the American Society for Information Science and Technology, 61(2): 424-427. 